\title{
Introducción al estudio de la transmisión de calor a través de cerramientos en régimen de temperatura variable periódicamente*
}

A. GARCIA ARROYO Licenciado en Ciencias Fisicos

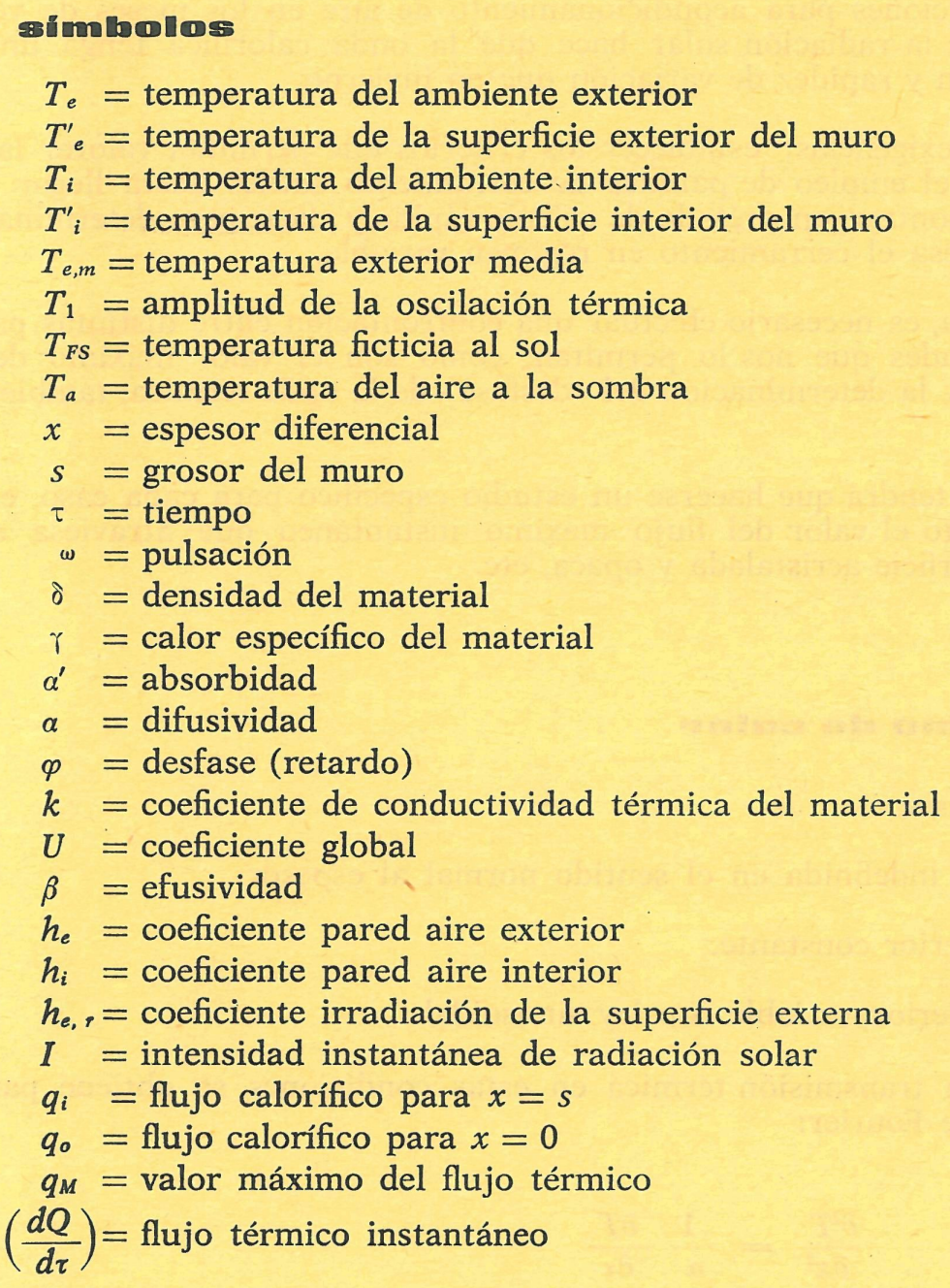

$311-11$

\section{simopers}

Como quiera que, en el estudio de la transmisión de calor a través de muros de cerramiento, no podemos prescindir del hecho de que la temperatura externa varíe periódicamente en función del tiempo, se efectúa aqui el cálculo teórico del flujo máximo de calor que atraviesa un muro de habitación, considerando la amortiguación y el desfase con que la onda calorífica llega a la cara interior, realizando a continuación algunas aplicaciones numéricas.

Para ello hemos de considerar "a priori» algunas hipótesis simplificadoras que nos permitan un estudio racional y no excesivamente complicado del problema planteado, 10 que conseguimos sirviéndonos de las ecuaciones de Alford, Ryan y Urban.

- No se estudian aquí paneles compuestos. 


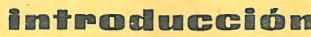

El estudio del comportamiento térmico de los muros de cerramiento en régimen de temperatura variable está tomando cada día un mayor interés, sobre todo a la vista del incremento que está experimentando el empleo de paredes ligeras con muy baja inercia térmica en la moderna construcción, así como en el uso de instalaciones de acondicionamiento de aire en los meses estivales, lo que condiciona a éstos a enfrentarse con unas oscilaciones térmicas mucho más amplias y rápidas que en invierno.

Desde el punto de vista del proyecto de una instalación acondicionadora de aire, se manifiesta, con lo antes dicho, la necesidad de recurrir a unos métodos de cálculo para la determinación de las características térmicas con las que ha de enfrentarse la planta, y particularmente en lo concerniente a la cantidad de calor intercambiada entre los ambientes externo e interno, mucho más exactos que los suministrados por la relación de Peclet, en la cual han de introducirse una serie de hipótesis no siempre aceptables, tales como la independencia en el tiempo de la temperatura de los ambientes a ambos lados de la pared.

En definitiva, la necesidad de un estudio más exacto y concienzudo de las características térmicas de las paredes externas en régimen de temperatura variable se debe a dos razones fundamentales:

a) al empleo, cada vez más extendido, de paredes ligeras con poca inercia térmica en relación con los muros tradicionales;

b) a la introducción de instalaciones para acondicionamiento de aire en los meses de verano en los que el efecto de la radiación solar hace que la onda calorífica tenga una mayor amplitud de oscilación y rapidez de variación que en invierno.

De aquí derivan las siguientes exigencias: establecer los criterios que permitan valorar las consecuencias que se deducen del empleo de paredes de cerramiento sutiles hasta llegar a una clasificación de éstos, y encontrar un método de cálculo rápido y eficaz para determinar la cantidad de calor que atraviesa el cerramiento en régimen variable.

En cuanto a la primera exigencia, es necesario efectuar una confrontación entre distintas paredes, estableciendo las magnitudes que nos lo permitan, como son el valor máximo del flujo del calor que la atraviesa y la determinación del «desfase» de la onda térmica, también llamado "retardo» $(\varphi)$.

Respecto a la segunda exigencia, tendrá que hacerse un estudio específico para cada caso, en el que habrá que considerar tanto el valor del flujo máximo instantáneo que atraviesa el muro como su orientación, superficie acristalada y opaca, etc.

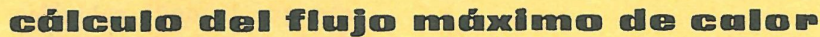

Se admiten las siguientes hipótesis:

1. Pared plana, homogénea e indefinida en el sentido normal al espesor.

2. ${ }^{2}$ Temperatura del flúido interior constante.

3. ${ }^{a}$ Temperatura del flúido exterior variable con ley sinusoidal.

La solución del problema de la transmisión térmica en estas condiciones se obtiene partiendo de la ecuación general de Fourier:

$$
\frac{\partial^{2} T}{\partial x^{2}}=\frac{1}{a} \frac{\partial T}{\partial \tau}
$$


En los límites, la solución será:

$$
\begin{aligned}
& q_{i}=-k\left(\frac{\partial T}{\partial x}\right)_{x=s}=h_{i}\left(T_{i}^{\prime}-T_{i}\right) \\
& q_{o}=-k\left(\frac{\partial T}{\partial x}\right)_{x=o}=h_{e}\left(T_{e}-T_{e}^{\prime}\right)
\end{aligned}
$$

Como quiera que la temperatura externa $\left(T_{e}\right)$ varía según una ley periódica sinusoidal (o al menos reducible a ella considerando las armónicas superiores de un desarrollo en serie de Fourier), la temperatura $\left(T^{\prime}{ }_{i}\right)$ de la cara interna de la pared también variará con ley sinusoidal de frecuencia igual a $T_{e}$, que nos lleva a escribir:

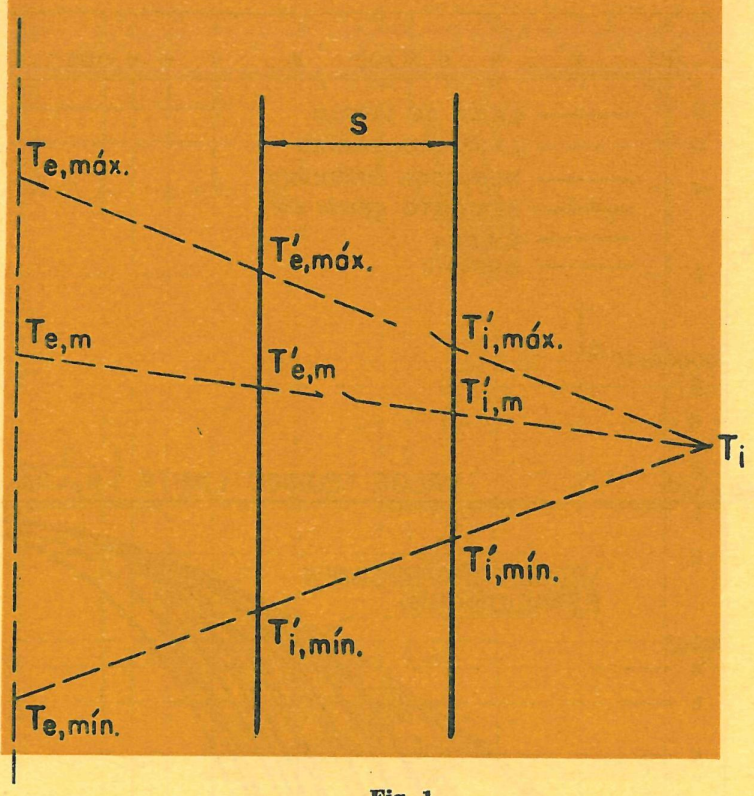

Fig. 1

$$
T^{\prime}{ }_{i}=T_{i}+\frac{1}{h_{i}} U\left(T_{e, m}-T_{i}\right)+\frac{V}{h_{i}} T_{1} \cos (\omega \tau-\varphi)
$$

en donde:

$$
\begin{gathered}
U=\frac{1}{\frac{1}{h_{e}}+\frac{1}{h_{i}}+\frac{s}{k}} ; \quad V=\frac{h_{e} h_{i}}{\beta k \sqrt{Y^{2}+Z^{2}}} ; \quad \beta=\sqrt{\frac{\omega \delta \gamma}{2 k}} \\
Y=\left[\frac{h_{e} h_{i}}{2 \beta^{2} k^{2}}+1\right] \cos (\beta s) \operatorname{Sh}(\beta s)+\left[\frac{h_{e} h_{i}}{2 \beta^{2} k^{2}}-1\right] \operatorname{sen}(\beta s) \operatorname{Ch}(\beta s)+\frac{h_{e} h_{i}}{\beta k} \cos (\beta s) \operatorname{Ch}(\beta s) \\
Z=\left[\frac{h_{e} h_{i}}{2 \beta^{2} k^{2}}+1\right] \operatorname{sen}(\beta s) \operatorname{Ch}(\beta s)-\left[\frac{h_{e} h_{i}}{2 \beta^{2} k^{2}}-1\right] \cos (\beta s) \operatorname{Sh}(\beta s)+\frac{h_{e} h_{i}}{\beta k} \operatorname{sen}(\beta s) \operatorname{Sh}(\beta s) \\
\varphi=\operatorname{Arctag} \frac{Z}{Y}
\end{gathered}
$$

El flujo térmico que pasa a través de la cara interna de la pared será:

$$
\left(\frac{d Q}{d \tau}\right)_{\max }=h_{i}\left(T_{i}^{\prime}-T_{i}\right)=U\left(T_{e, m}-T_{i}\right)+T_{1} V \cos (\omega \tau-\varphi)=q_{o}+q_{M} \cos (\omega \tau-\varphi)
$$

Es decir, que el flujo térmico instantáneo será la suma de una cantidad constante y otra variable periódicamente.

Por lo tanto:

$$
\frac{q_{M}}{T_{1} h_{e}}=\frac{h_{i}}{\beta k \sqrt{Y^{2}}+Z^{2}}=\frac{\frac{h_{i}}{h_{e}}}{\sqrt{\frac{\omega \delta \gamma k}{2 h_{e}^{2}}} \sqrt{Y^{2}+Z^{2}}}
$$

Dado que el flujo de calor depende solamente de los grupos $\omega \delta \gamma k$ y $s / k$, si lo representamos para distintos valores de esos grupos en diferentes materiales, llegamos a una serie de consecuencias notables para nuestro estudio: 


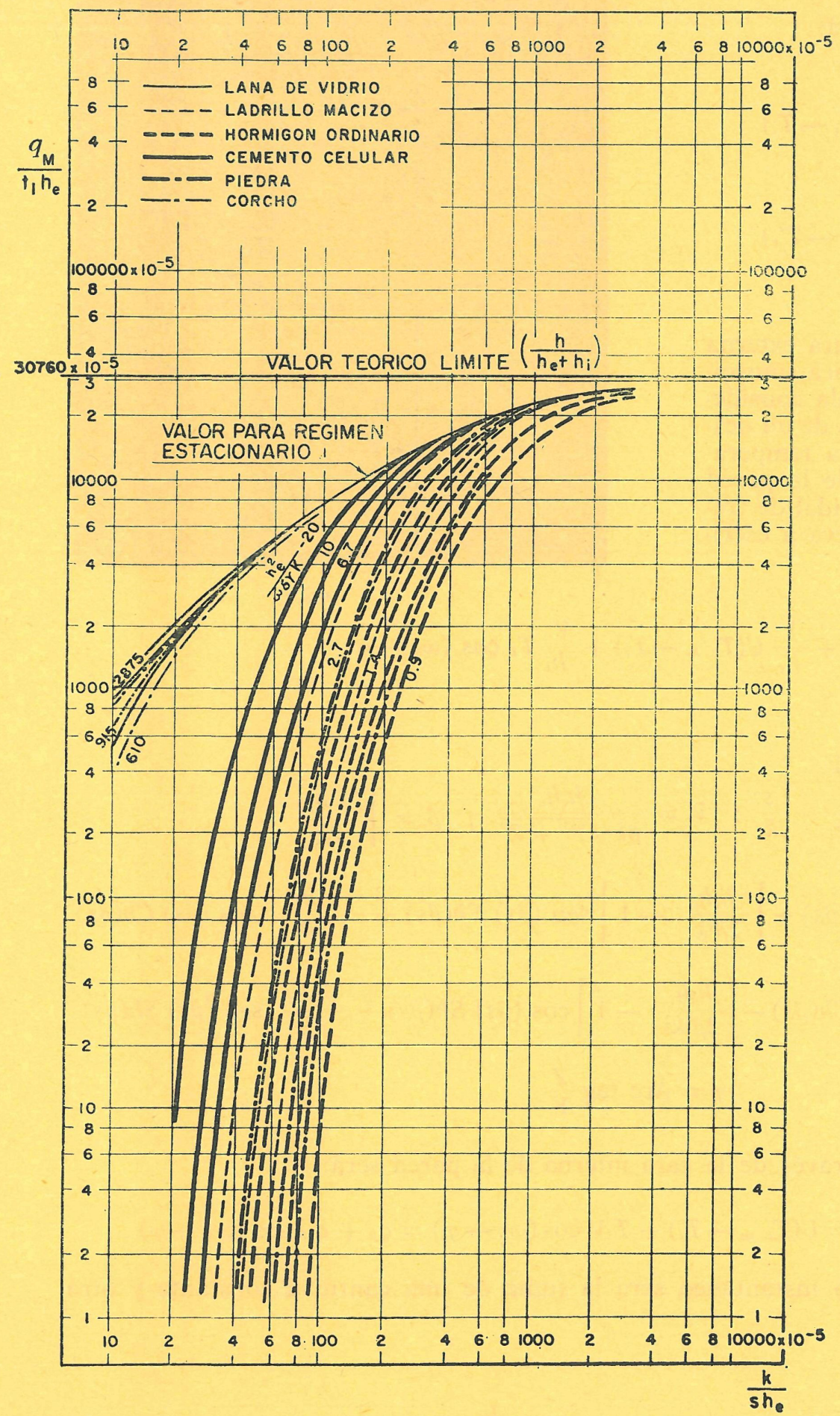

Fig. 2
Veamos las curvas de los siguientes materiales:

- lana de vidrio,

- ladrillo macizo,

- hormigón,

- cemento celular,

- piedra,

- corcho,

para sus espesores más frecuentes en la construcción, con los coeficientes $\delta, \gamma, k, h_{e}$ y $h_{i}$ de la tabla adjunta y los períodos de oscilación de 24,12 y 8 horas.

También hemos representado la curva correspondiente al régimen permanente definida por la relación:

$$
\frac{q}{\Delta T h_{e}}=\frac{1}{\frac{1}{h_{e}}+\frac{1}{h_{i}}+\frac{s}{k}}
$$

De la figura deducimos y confirmamos que:

1. Los materiales aislantes, como la lana de vidrio o el corcho, no tienen, prácticamente, efecto de inercia hasta que no se emplean con espesores superiores a 10 centímetros.

2. La independencia entre el valor máximo del flujo térmico $\left(q_{M}\right)$ y la efusividad $(k \delta \gamma)$ se manifiesta en todos los materiales cuando la relación entre el coeficiente de conductividad térmica y el espesor $(k / s)$ supera las $70 \mathrm{cal} /$ $/ \mathrm{m}^{2} \cdot \mathrm{h} \cdot{ }^{\circ} \mathrm{C}$. Por ejemplo en hormigón con espesores inferiores a 2 centímetros.

3. En las condiciones anteriores, la diferencia entre el valor de $q_{M}$ y el obtenido en régimen estacionario, con temperatura externa parecida a la amplitud de onda $\left(T_{1}\right)$ es inferior al 10 por 100 .

4. Cuanto mayor es el valor de la efusividad $(\delta \gamma k)$, su influencia es más notable.

Consideremos que los valores de los elementos $k, \delta$ y $\gamma$ han de ser lo más precisos posible. 


\begin{tabular}{|c|c|c|c|c|c|c|c|}
\hline $\begin{array}{l}066^{\prime} Z- \\
S L 9^{\prime} \varepsilon- \\
90 \nabla^{\prime} b- \\
00 L^{\prime} S- \\
09 Z^{\prime} 8- \\
L \forall Z^{\prime} \subseteq I- \\
9 \forall Z^{\prime} I Z- \\
L Z 0^{\prime} \subseteq \varepsilon-\end{array}$ & $\begin{array}{l}606^{\prime} 0 \\
S 86^{\prime} 0 \\
90 Z^{\prime} I \\
\tau L 9^{\prime} I \\
\varepsilon 0 \varepsilon^{\prime} Z \\
S \varepsilon Z^{\prime} D \\
Z 06^{\prime} \subseteq \\
0 \varepsilon L^{\prime} 6\end{array}$ & $\begin{array}{l}29 I^{\prime} 0 \\
\varepsilon 6 I^{\prime} 0 \\
6 E Z^{\prime} 0 \\
\text { †I } E^{\prime} 0 \\
6 S^{\prime} t^{\prime} 0 \\
2 \$ 8^{\prime} 0 \\
08 I^{\prime} I \\
97 \sigma^{\prime} I\end{array}$ & $\begin{array}{c}\ll \\
\ll \\
\ll \\
\ll \\
\ll \\
\ll \\
\ll \\
6 \nabla^{\prime} 0\end{array}$ & $\begin{array}{l}\ll \\
\ll \\
\ll \\
\ll \\
\ll \\
\ll \\
" \\
96\end{array}$ & $\begin{array}{c}\ll \\
\ll \\
\ll \\
\ll \\
\ll \\
\ll \\
\ll \\
\varepsilon 0^{\prime} 0\end{array}$ & $\begin{array}{l}8 I^{\prime} 0 \\
S I^{\prime} 0 \\
Z I^{\prime} 0 \\
60^{\prime} 0 \\
90^{\prime} 0 \\
80^{\prime} 0 \\
20^{\prime} 0 \\
10^{\prime} 0\end{array}$ & 'очріоว \\
\hline $\begin{array}{l}\varepsilon S L^{\prime} L I- \\
090^{\prime} \varepsilon Z- \\
6 t^{\prime} 9 Z- \\
\text { S00'IE- } \\
\angle S T^{\prime} L E- \\
66 Z^{\prime} L D- \\
8 S I^{\prime}+9-\end{array}$ & 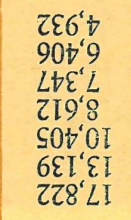 & $\begin{array}{l}986^{\prime} 0 \\
182^{\prime} I \\
697^{\prime} 1 \\
22 L^{\prime} I \\
180^{\prime} Z \\
829^{\prime} Z \\
+9 S^{\prime} \varepsilon\end{array}$ & $\begin{array}{l}\ll \\
\ll \\
\ll \\
\ll \\
\ll \\
\tau^{\prime} 0\end{array}$ & $\begin{array}{c}\ll \\
\ll \\
\ll \\
\ll \\
\ll \\
006 I\end{array}$ & $\begin{array}{l}\ll \\
" \\
" \\
" \\
" \\
" \\
Z^{\prime} I\end{array}$ & $\begin{array}{l}00^{\prime} I \\
Z L^{\prime} 0 \\
09^{\prime} 0 \\
8 b^{\prime} 0 \\
9 \mathcal{E}^{\prime} 0 \\
\forall Z^{6} 0 \\
Z I^{\prime} 0\end{array}$ & 'еxpo!d \\
\hline 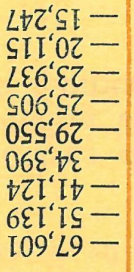 & $\begin{array}{l}S E Z^{\prime} \downarrow \\
88 S^{\prime} S \\
679^{\prime} 9 \\
96 I^{\prime} L \\
80 Z^{\prime} 8 \\
\varepsilon S S^{\prime} 6 \\
\varepsilon Z D^{\prime} I I \\
\text { SOZ'†I } \\
8 L L^{\prime} 8 I\end{array}$ & 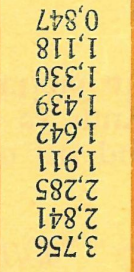 & $\begin{array}{c}\ll \\
\ll \\
\ll \\
\ll \\
\ll \\
\ll \\
\ll \\
\ll \\
z \tau^{\prime} 0\end{array}$ & $\begin{array}{l}\ll \\
\ll \\
\ll \\
\ll \\
\ll \\
\ll \\
\ll \\
\ll \\
008\end{array}$ & $\begin{array}{c}" \\
" \\
" \\
" \\
" \\
" \\
" \\
" \\
\varsigma \varepsilon^{\prime} 0\end{array}$ & $\begin{array}{l}S \varepsilon^{\prime} 0 \\
S Z^{\prime} 0 \\
0 Z^{\prime} 0 \\
8 I^{\prime} 0 \\
S I^{\prime} 0 \\
Z I^{\prime} 0 \\
60^{\prime} 0 \\
90^{\prime} 0 \\
\varepsilon 0^{\prime} 0\end{array}$ & 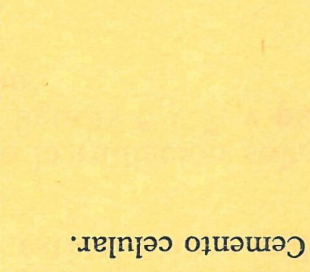 \\
\hline 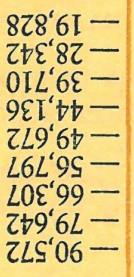 & $\begin{array}{l}80 S^{\prime} S \\
\varepsilon L 8^{\prime} L \\
\text { IEO'II } \\
09 Z^{\prime} Z I \\
86 L^{\prime} \varepsilon I \\
L L L^{\prime} S I \\
\text { 6It'8I } \\
\varepsilon Z I^{\prime} Z Z \\
6 S I^{\prime} S Z\end{array}$ & $\begin{array}{l}Z 0 I^{\prime} I \\
S L S^{\prime} I \\
90 Z^{\prime} Z \\
Z S t^{\prime} Z \\
09 L^{\prime} Z \\
S S I^{\prime} \varepsilon \\
\forall 89^{\prime} \varepsilon \\
S Z t^{\prime} t \\
Z E 0^{\prime} S\end{array}$ & $\begin{array}{c}\ll \\
\ll \\
\ll \\
\ll \\
\ll \\
\ll \\
\ll \\
\ll \\
I Z^{\prime} 0\end{array}$ & 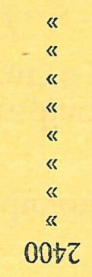 & $\begin{array}{l}\text { " } \\
" \\
" \\
" \\
" \\
" \\
" \\
" \\
\text { I'I }\end{array}$ & $\begin{array}{l}08^{\prime} 0 \\
0 S^{\prime} 0 \\
0 \mathcal{E}^{\prime} 0 \\
S Z^{\prime} 0 \\
0 Z^{\prime} 0 \\
S I^{\prime} 0 \\
0 I^{\prime} 0 \\
S 0^{\prime} 0 \\
Z O^{\prime} 0\end{array}$ & 'от̣еụp. \\
\hline 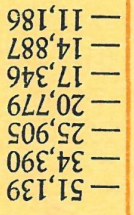 & $\begin{array}{l}\angle 0 I^{\prime} E \\
S E I^{\prime} b \\
8 I 8^{\prime} \downarrow \\
Z L L^{\prime} S \\
96 I^{\prime} L \\
E S S^{\prime} 6 \\
S 0 Z^{\prime} \triangleright I\end{array}$ & $\begin{array}{l}I Z 9^{\prime} 0 \\
L Z 8^{\prime} 0 \\
t 96^{\prime} 0 \\
t S I^{\prime} I \\
6 \varepsilon t^{\prime} I \\
I I 6^{\prime} I \\
I \forall 8^{\prime} Z\end{array}$ & 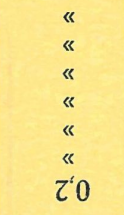 & $\begin{array}{c}\ll \\
\ll \\
\ll \\
\ll \\
\ll \\
\ll \\
00 L I\end{array}$ & $\begin{array}{l}" \\
" \\
\ll \\
" \\
" \\
" \\
L \prime 0\end{array}$ & $\begin{array}{l}00^{\prime} I \\
Z L^{\prime} 0 \\
09^{\prime} 0 \\
8 t^{\prime} 0 \\
9 \mathcal{E}^{\prime} 0 \\
\nabla Z^{\prime} 0 \\
Z I^{\prime} 0\end{array}$ & ozỊeu olI!xpeT \\
\hline $\begin{array}{l}Z I 6^{\prime} Z- \\
\text { SLt'E- } \\
90 \mathcal{E}^{\prime} t- \\
6 S 9^{\prime} S- \\
\text { SSZ'8- } \\
\angle \forall Z^{\prime} S L- \\
9 \forall Z^{\prime} I Z- \\
\text { LZO'SE- }\end{array}$ & $\begin{array}{l}608^{\prime} 0 \\
S 96^{\prime} 0 \\
96 I^{\prime} I \\
Z L S^{\prime} I \\
\varepsilon 6 Z^{\prime} Z \\
S E Z^{\prime} D \\
Z 06^{\prime} S \\
0 E L^{\prime} 6\end{array}$ & $\begin{array}{l}z 9 I^{\prime} 0 \\
\varepsilon 6 I^{\prime} 0 \\
6 \varepsilon Z^{\prime} 0 \\
t I \varepsilon^{\prime} 0 \\
6 S t^{\prime} 0 \\
\angle \forall 8^{\prime} 0 \\
08 I^{\prime} I \\
9 b 6^{\prime} I\end{array}$ & $\begin{array}{c}\ll \\
\ll \\
\ll \\
« \\
\ll \\
\ll \\
\tau^{\prime} 0\end{array}$ & $\begin{array}{l}\ll \\
\ll \\
" \\
" \\
\ll \\
" \\
" \\
08\end{array}$ & $\begin{array}{c}\ll \\
\ll \\
\ll \\
\ll \\
\ll \\
\ll \\
\ll \\
\varepsilon 0^{\prime \prime} 0\end{array}$ & $\begin{array}{l}8 I^{\prime} 0 \\
S I^{\prime} 0 \\
Z I^{\prime} 0 \\
60^{\prime} 0 \\
90^{\prime} 0 \\
\varepsilon 0^{\prime} 0 \\
Z 0^{\prime} 0 \\
I 0^{\prime} 0\end{array}$ & -oixpis op euet \\
\hline$x p u\left(\frac{2 p}{\partial p}\right)$ & ${ }^{x p u}\left(\frac{2 p}{\partial p}\right)$ & $\mathbf{n}$ & 6 & 8 & Y & $\mathbf{S}$ & \\
\hline \multicolumn{2}{|c|}{ 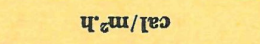 } & 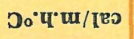 & 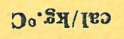 & gur/gY & $D_{0} \cdot 4 \cdot u / /$ Ieכ & us & \\
\hline
\end{tabular}

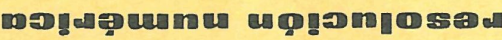

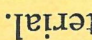

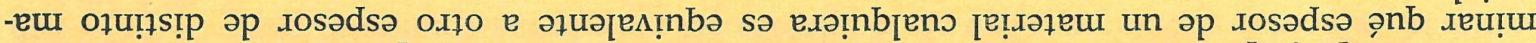

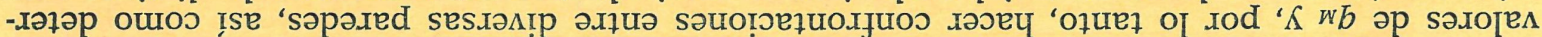

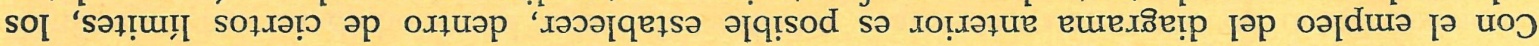


Confirmaremos lo dicho en el apartado anterior realizando una serie de determinaciones de $\left(\frac{d Q}{d \tau}\right)_{\text {max }}$ para diferentes paredes en las estaciones de estío e invierno, lo que haremos mediante la ecuación de Alford, Ryan y Urban, ya conocida:

$$
\left(\frac{d Q}{d \tau}\right)_{\max }=U\left(T_{e, m}-T_{i}\right)+T_{1} \cos (\omega \tau-\varphi) .
$$

Elegimos dos días tipo, uno de verano y otro de invierno, cuyas condiciones extremas vemos en las figuras 3 y 4 . La temperatura interna permanece constante en todos los casos, pues su-

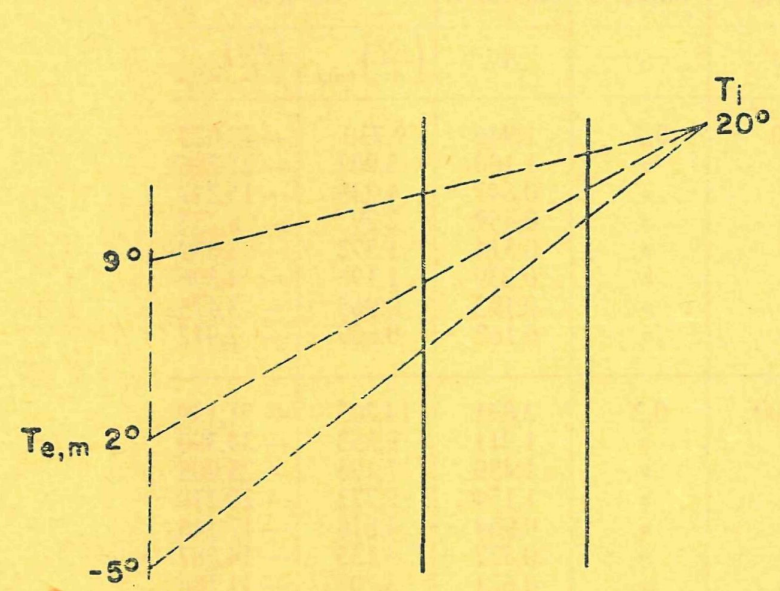

Fig. 3

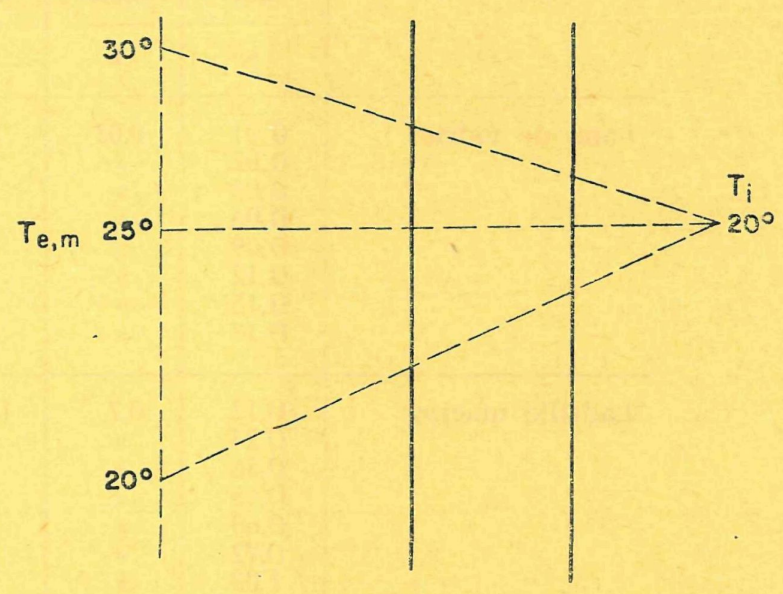

Fig. 4

ponemos una habitación provista de acondicionamiento de aire en la que la temperatura no cambia de $20^{\circ} \mathrm{C}$.

En invierno consideramos que no existe radiación solar, o al menos es despreciable. La variación diaria de temperatura sigue una curva del tipo a la dibujada en la figura 5.

En dicha curva vemos que la diferencia $\left(T_{e}, m-T_{i}\right)$ es lo suficientemente grande (en algunos casos, decenas de grados) como para que la máxima aportación de calor proceda del término constante en el tiempo $U\left(T_{e, m}-T_{i}\right)$, mientras que en la estación estival ocurre todo lo contrario.

En los meses de verano no podemos despreciar el fenómeno de la radiación solar, ya que su aportación calorífica es muy importante, como vemos en las figuras 6 y 7 , y para determinar la temperatura ficticia al sol lo haremos según la ecuación:

$$
T_{F S}=T_{a}+\frac{a^{\prime} I}{h_{e}}+\left(t_{r}-t_{a}\right) \frac{h_{e} r}{h_{e}}
$$

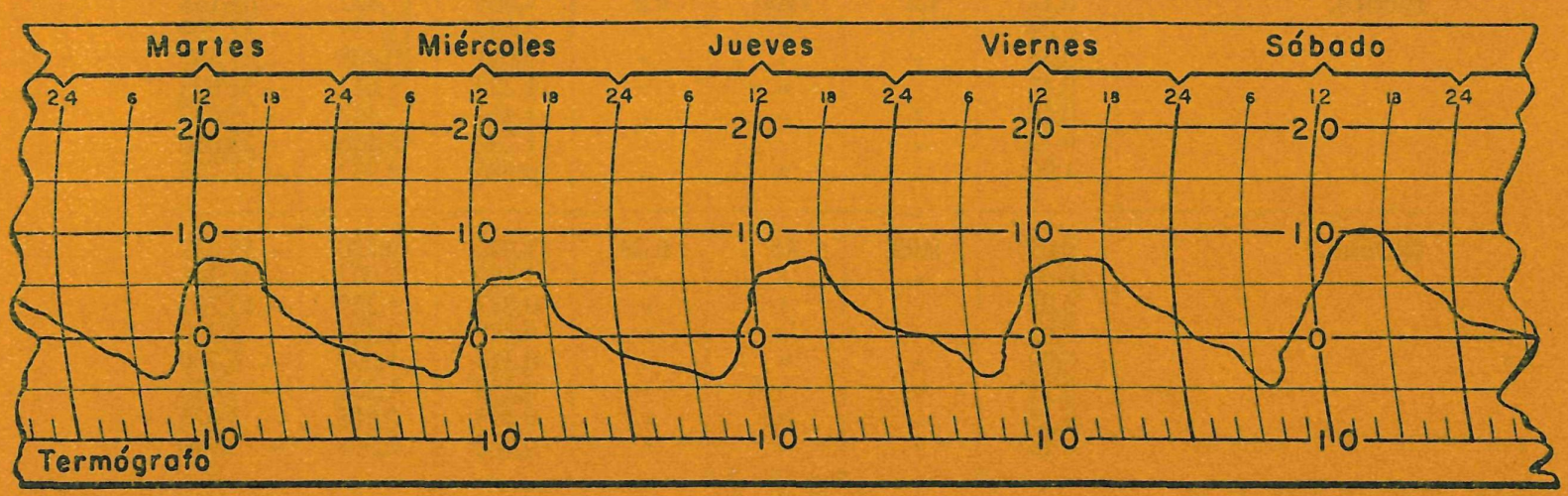


en la que:

$I$ es la intensidad de irradiación directa y difusa recibida por una pared que tiene una absorbidad $\alpha^{\prime}$ y un coeficiente de irradiación superficial $h_{e}, r$;

$T_{a}$ es la temperatura del aire sin considerar el fenómeno de la radiación solar.

Con estos datos y los de la tabla I calculamos el valor del flujo térmico máximo en los dos períodos establecidos, cuyas funciones representativas de la temperatura exterior son:

$$
\begin{aligned}
& T_{e}=-18-7 \cos (15 \tau-\varphi) \\
& T_{e}=5-5 \cos (15 \tau-\varphi)
\end{aligned}
$$

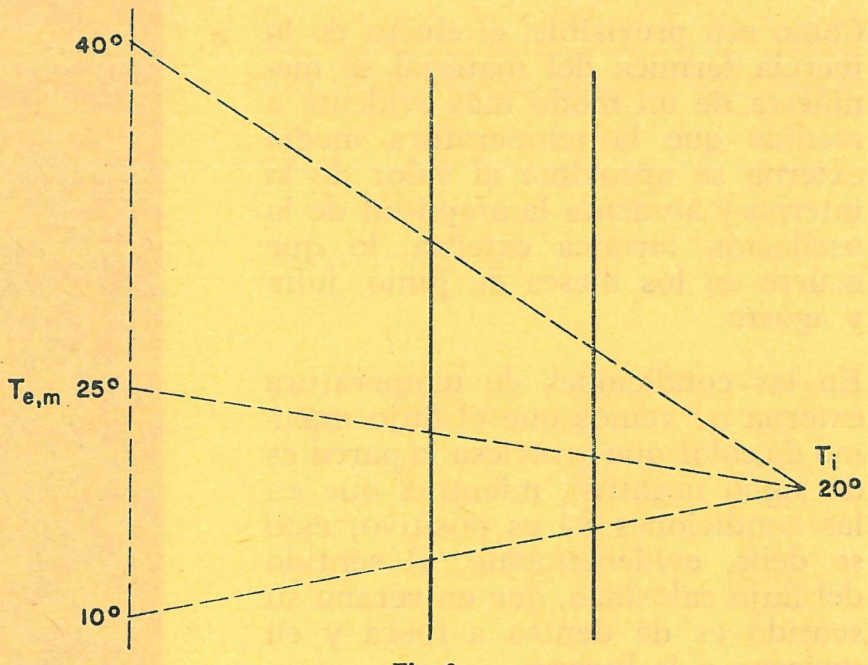

Fig. 6

Las curvas de las figuras 8 y 9 las obtenemos llevando al sistema cartesiano los valores de la tabla I, en el que dibujamos el flujo térmico máximo en función del espesor.

Observando estos gráficos vemos que en el primero, por ejemplo, el mismo flujo específico máximo se obtiene para un panel de $2 \mathrm{~cm}$ de espesor de lana de vidrio que para otro de $25 \mathrm{~cm}$ de cemento celular, ó $12 \mathrm{~cm}$ de cemento celular y $24 \mathrm{~cm}$ de ladrillo macizo. Del mismo modo, en las condiciones de verano vemos que el mismo flujo atravesará por un panel de $32 \mathrm{~cm}$ de ladrillo macizo que por $50 \mathrm{~cm}$ de otro de hormigón ordinario.

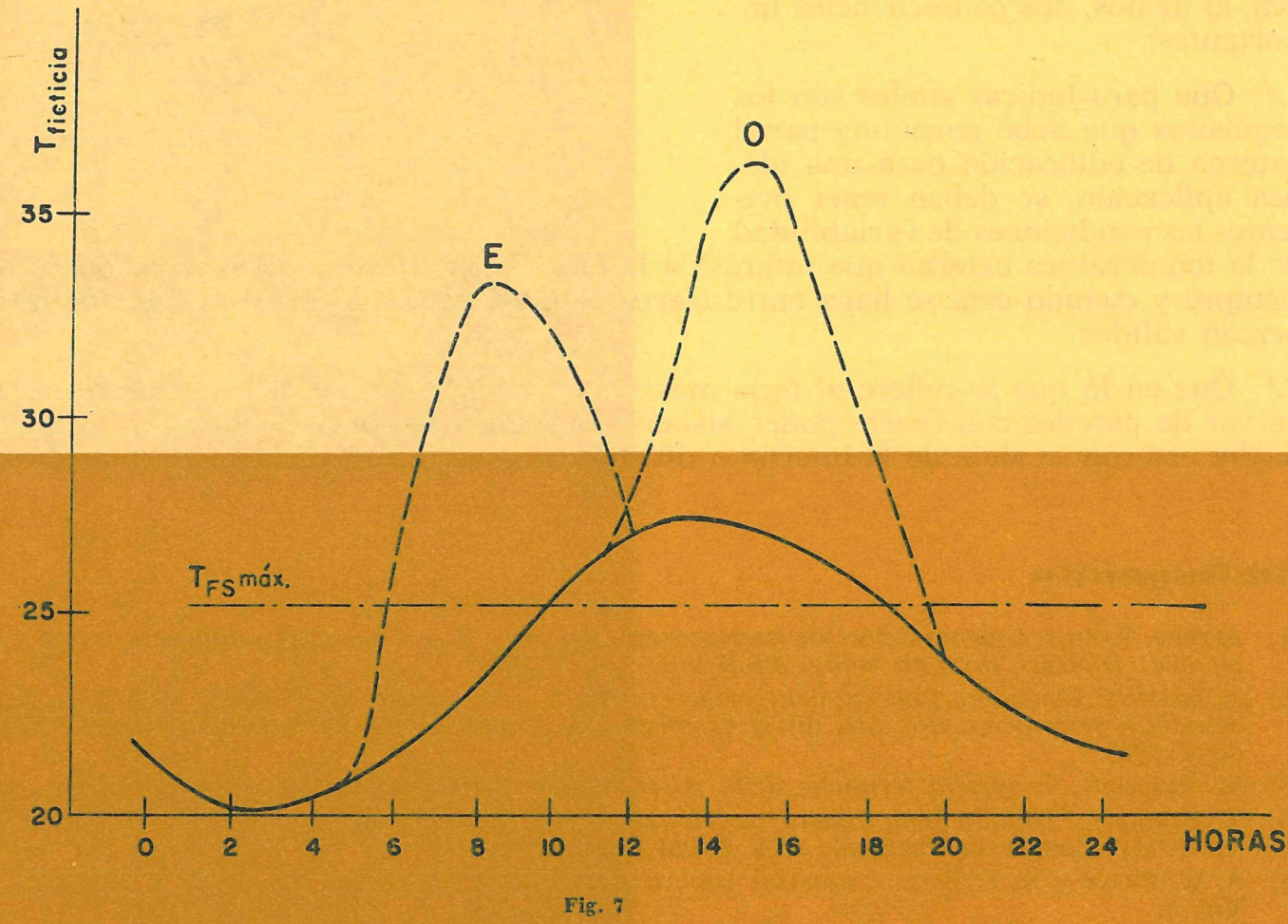


Como era previsible, el efecto de la inercia térmica del material se manifiesta de un modo más evidente a medida que la temperatura media externa se aproxima al valor de la interna y aumenta la amplitud de la oscilación térmica externa, lo que ocurre en los meses de junio, julio y agosto.

En las condiciones de temperatura externa a) vemos que el flujo máximo de calor que atraviesa la pared es de signo negativo, mientras que en las condiciones $b$ ) es positivo; esto se debe, evidentemente, al sentido del flujo calorífico, que en verano su sentido es de dentro a fuera y en invierno a la inversa.

Observemos en los gráficos los valores tan bajos obtenidos para materiales aislantes (corcho, lana de vidrio), los cuales tienen unas propiedades térmicas casi idénticas; ello nos afianza en la validez de este método por su correspondencia con la práctica.

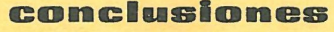

De todo lo dicho más arriba se deducen, al menos, dos consecuencias importantes:

1. ${ }^{\text {a }}$ Que para indicar cuáles son los requisitos que debe tener una pared externa de edificación para una idónea aplicación, se deben tener presentes las condiciones de variabilidad de la temperatura externa que interesa a la pared. Esto no impide la creación de una norma, siempre y cuando ésta se haga entre ciertos límites para que los criterios adoptados permanezcan válidos.

2. ${ }^{\text {a }}$ Que en lo que se refiere al flujo máximo, el empleo de paredes con gran inercia térmica en vez de paredes con fuerte poder aislante es menos importante en cuanto la temperatura media externa se aleje de la interna o disminuya la amplitud de la oscilación térmica.

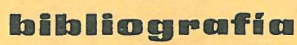

(1) ALFORD, RYAN y URBAN: Effect of heat storage and variation in outdoor temperature and solar intensity on heat transfer through walls. A.S.H.V.E., vol. 45, 1939.

(2) A. FanTINI: Considerazioni sul comportamento delle pareti sterne delli edifici in regime di temperatura variàbile periodicamente. Atti dil 3. ${ }^{\circ}$ Convegno Nazionale del Riscaldamento e della Ventilazione. Pàdova, 1963.

(3) M. PARIBEnI: Proprietà termiche delle pareti sottili. La Termotècnica, núm. 10, 1963.

(4) I. BARDUCcI: Método semplificato per il càlcolo dell'attenuazione di pareti in regime tèrmico periòdico. XVIII Congresso Nazionale A. T. I. Palermo, 1963.

(5) A. W. PRATt y E. F. Ball: Transient cooling of a heated enclousure. I. N. T. J. Heat Mass Transfer. Vol. 6. 


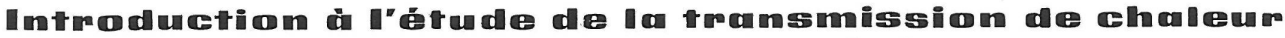

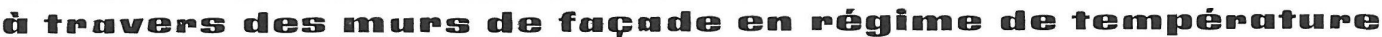

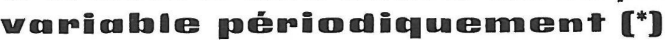

A. García Arroyo, licencié ès Sciences Physiques

Pour l'étude de la transmission de chaleur à travers des murs de façade on ne peut négliger le fait que la température extérieure varie périodiquement en fonction du temps. Pour cette raison, le calcul théorique du flux maximal de chaleur qui traverse un mur d'habitation est effectué ici, en considérant l'amortissement et le déphasage avec lesquels l'onde calorifique arrive à la face intérieure. Des applications numériques sont réalisées ensuite.

On doit considérer «a priori» quelques hypothèses simplificatrices qui permettent une étude rationnelle et non excessivement compliquée du problème posé, ce qui est réalisé à l'aide des équations d'Alford, Ryan et Urban.

${ }^{*}$ Les panneaux composés ne sont pas étudiés ici.

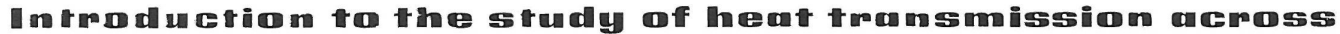

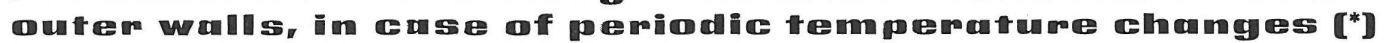

A. García Arroyo, Graduate in physics

In the study of heat transmission across outer walls, it is not possible to avoid the fact that external temperature varies periodically. Hence in this paper a theoretical calculation is made of the maximum heat flow across a wall, taking into account the damping and retardation acting on the heat wave, as it reaches the inner face of the wall. A number of practical numerical applications are given.

A number of a priori simplifying hypotheses have been made, which enable a rational and non too complex study of the problem to be made. This has been possible by the use of the Alford, Ryan and Urban equations.

(*) Compound panels have not been considered.

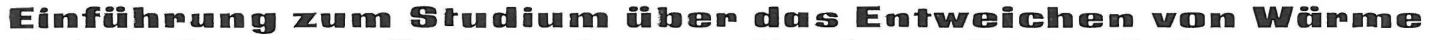

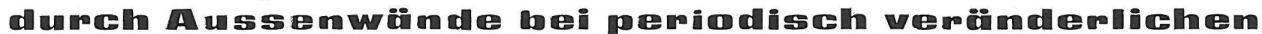

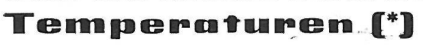

A. García Arroyo, Dipl.-Physiker

Da man bei dem Studium der Wärmeübertragung durch Aussewände die Tatsache nicht übersehen darf, dass die Temperatur sich auf Grund der Witterung periodisch ändert, wird hier das Entweichen von Wärme durch eine Zimmerwand theoretisch berechnet, wobei man gleichzeitig die Dämpfung und Phasenverschiebung, mit der die Wärmewelle auf die Innenseite gelangt, in Betracht zieht. Danach werden einige Zahlenbeispiele gegeben.

Dazu müssen im voraus einige Vereinfachungshypothesen aufgestellt werden, die ein rationelles und nicht zu kompliziertes Studium des gestellten Problems ermöglichen. Dies erreicht man durch die Anwendung der Gleichungen von Alford, Ryan und Urban.

(*) Zusammengesetzte Panele werden hier nicht behandelt. 